\title{
Analysis of weighted Laplacian and applications to Ricci solitons
}

\author{
Ovidiu Munteanu And Jiaping Wang
}

\begin{abstract}
We study both function theoretic and spectral properties of the weighted Laplacian $\Delta_{f}$ on complete smooth metric measure space $\left(M, g, \mathrm{e}^{-f} d v\right)$ with its Bakry-Émery curvature $\mathrm{Ric}_{f}$ bounded from below by a constant. In particular, we establish a gradient estimate for positive $f$-harmonic functions and a sharp upper bound of the bottom spectrum of $\Delta_{f}$ in terms of the lower bound of $\operatorname{Ric}_{f}$ and the linear growth rate of $f$. We also address the rigidity issue when the bottom spectrum achieves its optimal upper bound under a slightly stronger assumption that the gradient of $f$ is bounded.

Applications to the study of the geometry and topology of gradient Ricci solitons are also considered. Among other things, it is shown that the volume of a noncompact shrinking Ricci soliton must be of at least linear growth. It is also shown that a nontrivial expanding Ricci soliton must be connected at infinity provided its scalar curvature satisfies a suitable lower bound.
\end{abstract}

\section{Introduction}

In our previous paper [20], we have studied some function theoretic and spectral properties of the weighted Laplacian on a smooth metric measure space with nonnegative Bakry-Émery curvature. We have also applied the results to conclude a nontrivial steady gradient Ricci soliton must be connected at infinity. The purpose of this sequel to [20] is two-fold. The first is to continue our study of the weighted Laplacian on a smooth metric measure space, now under the more general assumption that its Bakry-Émery curvature is bounded from below by a negative constant. The second is to demonstrate that the results and techniques from such study lead to geometric and topological information of shrinking and expanding gradient Ricci solitons.

Recall that a smooth metric measure space, denoted by $\left(M, g, \mathrm{e}^{-f} d v\right)$ throughout the paper, is a Riemannian manifold $(M, g)$ together with a weighted volume form $\mathrm{e}^{-f} d v$, where $f$ is a smooth function on $M$ and $d v$ 
the volume element induced by the Riemannian metric $g$. The associated weighted Laplacian $\Delta_{f}$ is given by

$$
\Delta_{f} u:=\Delta u-\langle\nabla f, \nabla u\rangle
$$

which is a self-adjoint operator on the space of square integrable functions on $M$ with respect to the measure $\mathrm{e}^{-f} d v$. A function $u$ is called $f$-harmonic if $\Delta_{f} u=0$. It is easy to see that $f$-harmonic functions are characterized as the critical points of the weighted Dirichlet energy $\int_{M}|\nabla u|^{2} \mathrm{e}^{-f} d v$.

The Bakry-Émery curvature Ric $f$ associated to smooth metric measure space $\left(M, g, \mathrm{e}^{-f} d v\right)$ is defined [1] by

$$
\operatorname{Ric}_{f}:=\operatorname{Ric}+\operatorname{Hess}(f) \text {, }
$$

where Ric denotes the Ricci curvature of $(M, g)$ and Hess $(f)$ the Hessian of $f$.

The weighted Laplacian and the Bakry-Émery curvature are natural objects in the geometric analysis. The most significant and interesting case of our concern here is the so-called gradient Ricci solitons. Recall a complete manifold $(M, g)$ is a gradient Ricci soliton if the equation $\operatorname{Ric}_{f}=\lambda g$ holds for some function $f$ and scalar $\lambda$. The soliton is called expanding, steady and shrinking, accordingly, if $\lambda<0, \lambda=0$ and $\lambda>0$. It is customary to normalize the constant $\lambda \in\{-1 / 2,0,1 / 2\}$ by scaling the metric $g$. As suggested by the name, the gradient Ricci solitons arise from the study of Ricci flows, particularly from the blow up analysis of the singularities of the Ricci flows [11]. It is thus a central issue in the study of Ricci flows to understand and classify gradient Ricci solitons. Note that the Ricci soliton equation $\operatorname{Ric}_{f}=\lambda g$ reduces to the Einstein equation $\mathrm{Ric}=\lambda g$ when $f$ is a constant function. So the soliton equation is also of own interest as a geometric partial differential equation. We refer the readers to the book [9] for more information on gradient Ricci solitons.

The Bakry-Émery curvature is closely related to the weighted Laplacian as indicated by the following Bochner type identity.

$$
\Delta_{f}|\nabla u|^{2}=2|\operatorname{Hess}(u)|^{2}+2\left\langle\nabla u, \nabla \Delta_{f} u\right\rangle+2 \operatorname{Ric}_{f}(\nabla u, \nabla u) .
$$

This is of course very much in parallel to how the Ricci curvature is related to the Laplacian on a complete manifold. Taking this point of view, in the first part of the paper, we will develop some analogous results to the Laplacian for the weighted Laplacian under the assumption that the Bakry-Émery curvature is bounded from below. However, we would like to point out that 
unlike the classical case on the analysis of the Laplacian, the function $f$ enters into play in the behavior of the weighted Laplacian $\Delta_{f}$. The assumptions on $f$ dictate both the conclusions and the level of technical difficulties involved in the arguments. As in [20], we continue to assume that $f$ grows at most linearly, that is,

$$
|f|(x) \leq \alpha r(x)+\beta
$$

for some constants $\alpha \geq 0$ and $\beta \geq 0$, where $r(x):=d(p, x)$ is the geodesic distance to a fixed point $p$ in $M$. The linear growth rate $a$ of $f$ is then defined to be the infimum over all such $\alpha$.

Also, as indicated, we assume that $\mathrm{Ric}_{f}$ is bounded below by a negative constant. After a suitable scaling of the metric, we may in fact assume $\operatorname{Ric}_{f} \geq-(n-1)$, where $n$ is the dimension of $M$.

Our first result is a gradient estimate for positive $f$-harmonic functions on $\left(M, g, \mathrm{e}^{-f} d v\right)$.

Theorem 1.1. Let $\left(M, g, \mathrm{e}^{-f} d v\right)$ be a smooth metric measure space of dimension $n$ with $\operatorname{Ric}_{f} \geq-(n-1)$. Assume that there exists constant $a>0$ such that the oscillation of $f$ over the unit ball $B_{x}(1)$ for any $x \in M$ satisfies

$$
\sup _{y \in B_{x}(1)}|f(y)-f(x)| \leq a
$$

Then there exists a constant $C(n, a)$ depending only on $n$ and a such that for any $u>0$ with $\Delta_{f} u=0$ we have

$$
|\nabla \log u| \leq C(n, a)
$$

We remark that the assumption on $f$ in Theorem 1.1 is satisfied, for example, if $|\nabla f| \leq a$ or if $f$ is bounded on $M$. In the case that $|\nabla f| \leq a$, the result can be proved more or less following the classical argument of Yau [30] via the aforementioned Bochner identity. The reference [33] is a good source for many other similar results concerning the weighted Laplacian, under various curvature bounds. If $\operatorname{Ric}_{f} \geq 0$, then the result has been proved in [20]. In fact, the following stronger conclusion holds.

$$
|\nabla \log u| \leq C(n) a
$$

Our second result concerns the bottom spectrum of the weighted Laplacian $\Delta_{f}$. Let $\lambda_{1}\left(\Delta_{f}\right):=\inf \operatorname{Spec}\left(-\Delta_{f}\right)$. Then the variational 
characterization for $\lambda_{1}\left(\Delta_{f}\right)$ implies that

$$
\lambda_{1}\left(\Delta_{f}\right)=\inf _{\phi \in C_{0}^{\infty}(M)} \frac{\int_{M}|\nabla \phi|^{2} \mathrm{e}^{-f} d v}{\int_{M} \phi^{2} \mathrm{e}^{-f} d v} .
$$

In the case $\operatorname{Ric}_{f} \geq 0$, it was shown in [20] that $\lambda_{1}\left(\Delta_{f}\right)$ has an optimal upper bound of the form $\lambda_{1}\left(\Delta_{f}\right) \leq \frac{1}{4} a^{2}$, where $a$ is the linear growth rate of $f$. Here is a more general result.

Theorem 1.2. Let $\left(M^{n}, g, \mathrm{e}^{-f} d v\right)$ be a complete smooth metric measure space with $\operatorname{Ric}_{f} \geq-(n-1)$. Then we have

$$
\lambda_{1}\left(\Delta_{f}\right) \leq \frac{1}{4}(n-1+a)^{2},
$$

where $a \geq 0$ is the linear growth rate of $f$. In particular, if $f$ is of sublinear growth, then the bottom spectrum of $\Delta_{f}$ satisfies the following sharp upper bound

$$
\lambda_{1}\left(\Delta_{f}\right) \leq \frac{(n-1)^{2}}{4} .
$$

This result is consistent with Cheng's well-known estimate [3] in the case $f$ is constant, which says that $\lambda_{1}(M)$ is bounded above by $\frac{(n-1)^{2}}{4}$. This incidentally indicates our estimate is sharp. We remark that under the stronger assumption that $|\nabla f| \leq a$, the result has also been established in $[27]$.

Motivated by the work of $\mathrm{Li}$ and the second author in [16-19] and our generalization in $[20]$ to the case of weighted Laplacian with $\operatorname{Ric}_{f} \geq 0$, we study the structure of manifolds on which $\lambda_{1}\left(\Delta_{f}\right)$ achieves its maximal value in the preceding estimate. Here, we need to impose a stronger assumption on $f$ that its gradient is bounded.

Theorem 1.3. Let $\left(M, g, \mathrm{e}^{-f} d v\right)$ be a complete smooth metric measure space of dimension $n \geq 3$ with $\operatorname{Ric}_{f} \geq-(n-1)$. Assume that $|\nabla f| \leq a$ on $M$ for some constant $a \geq 0$. If $\lambda_{1}\left(\Delta_{f}\right)=\frac{1}{4}(n-1+a)^{2}$, then either $M$ is connected at infinity or $f$ is constant and $M$ splits as a warped product $M=$ $\mathbb{R} \times N$ with $d s_{M}^{2}=d t^{2}+h^{2}(t) d s_{N}^{2}$, where $N$ is compact and the function $h(t)=\mathrm{e}^{t}$ if $n \geq 4$ and $h(t)=\mathrm{e}^{t}$ or $h(t)=\cosh t$ if $n=3$.

Let us point out that in the case $M$ is the warped product, its bottom spectrum has maximal value $\frac{(n-1)^{2}}{4}$. Let us also point out that this result has been independently proved by Su and Zhang in [27]. 
In the second part of this paper, we consider some applications of our study of the weighted Laplacian to gradient Ricci solitons. We first address the issue whether a nontrivial expanding gradient Ricci soliton must be connected at infinity. Recall that an expanding gradient Ricci soliton is a manifold $(M, g)$ such that $\operatorname{Ric}_{f}=-\frac{1}{2} g$ for some function $f$. It is known [24] that the scalar curvature $S \geq-\frac{n}{2}$ on an expanding gradient Ricci soliton.

We have the following result concerning this issue.

Theorem 1.4. Let $(M, g, f)$ be an expanding gradient Ricci soliton. Assume that $S \geq-\frac{n-1}{2}$ on $M$. Then either $M$ is connected at infinity or $M$ is isometric to the product $\mathbb{R} \times N$, where $N$ is a compact Einstein manifold and $\mathbb{R}$ the Gaussian expanding Ricci soliton.

Note that in the second case of $M$ being a cylinder, its scalar curvature $S=-\frac{n-1}{2}$. This somewhat explains why we impose such an assumption on $S$. However, at this point it is unclear to us whether the assumption is in fact superfluous for nontrivial expanding gradient Ricci solitons, although obviously there are Einstein manifolds with infinitely many ends.

As for the proof of Theorem 1.4, it does not follow directly from our preceding rigidity theorem. This is because for expanding gradient Ricci solitons, the potential function $f$ is never of linear growth unless it is trivial according to the following result.

Theorem 1.5. Let $(M, g, f)$ be a nontrivial expanding gradient Ricci soliton. Then for all $r>2$,

$$
\frac{1}{4} r^{2}-C r^{\frac{3}{2}} \sqrt{\ln r} \leq \sup _{\partial B_{p}(r)}(-f)(x) \leq \frac{1}{4} r^{2}+C r
$$

for some constant $C$.

At this point, it seems interesting to compare our result with the case of shrinking gradient Ricci solitons. It has been shown by Cao and Zhou [6] that

$$
\frac{1}{4}(r(x)-c)^{2} \leq f(x) \leq \frac{1}{4}(r(x)+c)^{2}
$$

on a nontrivial, noncompact, shrinking gradient Ricci soliton. We also point out that it was first observed in [24] that the gradient of the potential function $f$ must be unbounded for a nontrivial expanding gradient Ricci soliton.

Another issue we resolve here is about the volume growth lower bound for shrinking gradient Ricci solitons. As well known, volume growth rate 
is an important piece of geometric information. In [6], it was proved that the volume of a shrinking gradient Ricci soliton is at most of polynomial growth of order $n$, the dimension of the underlying manifold. Concerning the lower bound, when the Ricci curvature is bounded, then it is known [7] the volume grows at least linearly. The question whether this is the case for general shrinking gradient Ricci solitons has been posed to the authors by Huaidong Cao and Lei $\mathrm{Ni}$, respectively. We confirm this to be the case here. Note that this is sharp as shown by the cylinder examples. Indeed, for $M=\mathbb{R} \times N^{n-1}$, where $N^{n-1}$ is an Einstein manifold such that $\operatorname{Ric}_{N}=\frac{1}{2} g_{N}$ and $\mathbb{R}$ is the Gaussian shrinking soliton with potential function $f=\frac{1}{4}|x|^{2}$, the volume of $M$ grows linearly.

Theorem 1.6. Let $(M, g, f)$ be a noncompact shrinking gradient Ricci soliton. Then there exists a constant $C>0$ such that

$$
V\left(B_{p}(r)\right) \geq C r, \quad \text { for all } r>0 .
$$

The paper is organized as follows. In Section 2, we prove Theorem 1.2 after some discussions on Laplacian and volume comparison results. We then prove Theorem 1.1 in Section 3. In Section 4, we study the structure of manifolds with maximal bottom spectrum $\lambda_{1}\left(\Delta_{f}\right)$ and prove Theorem 1.3. In Section 5, we consider the expanding Ricci solitons and prove both Theorems 1.4 and 1.5. In the final Section 6, we deal with the shrinking Ricci solitons and prove Theorem 1.6.

We would like to thank Huai-Dong Cao for his interest and stimulating comments, which lead us to improve both Theorems 5.1 and 6.1.

\section{Volume comparison theorem}

In this section, following Wei and Wylie [28], we discuss Laplacian and volume comparison results by assuming a lower bound on the Bakry-Émery curvature tensor. As an immediate consequence, we obtain an upper bound estimate for the bottom spectrum of $\Delta_{f}$.

Let $\left(M, g, \mathrm{e}^{-f} d v\right)$ be a smooth metric measure space. Take any point $x \in$ $M$ and express the volume form in the geodesic polar coordinates centered at $x$ as

$$
\left.d V\right|_{\exp _{x}(r \xi)}=J(x, r, \xi) d r d \xi
$$


for $r>0$ and $\xi \in S_{x} M$, a unit tangent vector at $x$. It is well known that if $y \in M$ is another point such that $y=\exp _{x}(r \xi)$, then

$$
\Delta d(x, y)=\frac{J^{\prime}(x, r, \xi)}{J(x, r, \xi)} \text { and } \Delta_{f} d(x, y)=\frac{J_{f}^{\prime}(x, r, \xi)}{J_{f}(x, r, \xi)}
$$

where $J_{f}(x, r, \xi):=\mathrm{e}^{-f} J(x, r, \xi)$ is the $f$-volume form in the geodesic polar coordinates. For a set $\Omega$ we will denote by $V(\Omega)$ the volume of $\Omega$ with respect to the usual volume form $d v$, and $V_{f}(\Omega)$ the $f$-volume of $\Omega$.

Lemma 2.1. Let $\left(M, g, \mathrm{e}^{-f} d v\right)$ be a complete smooth metric measure space with $\operatorname{Ric}_{f} \geq-(n-1)$. Assume for some nonnegative constants $\alpha$ and $\beta$,

$$
|f|(x) \leq \alpha r(x)+\beta
$$

for $x \in M$. Then there exists a constant $C>0$ such that the volume upper bound

$$
V_{f}\left(B_{p}(R)\right) \leq C \mathrm{e}^{(n-1+\alpha) R}
$$

holds for all $R>0$.

Proof of Lemma 2.1. As discussed above, we write $\left.d V\right|_{\exp _{p}(r \xi)}=J(r, \xi) d r d \xi$ for $\xi \in S_{p} M$. Let $J_{f}(r, \xi)=\mathrm{e}^{-f(r, \xi)} J(r, \xi)$ be the corresponding weighted volume form. In the following, we will omit the dependence of these quantities on $\xi$. Along a minimizing geodesic starting from $p$, we have

$$
\left(\frac{J^{\prime}}{J}\right)^{\prime}(r)+\frac{1}{n-1}\left(\frac{J^{\prime}}{J}\right)^{2}(r)+\operatorname{Ric}\left(\frac{\partial}{\partial r}, \frac{\partial}{\partial r}\right) \leq 0,
$$

where the differentiation is with respect to the $r$ variable. Integrating this inequality from 1 to $r$ and using the assumption that

$$
\operatorname{Ric}\left(\frac{\partial}{\partial r}, \frac{\partial}{\partial r}\right)+f^{\prime \prime}(r) \geq-(n-1),
$$

we get

$$
\frac{J^{\prime}}{J}(r)+\frac{1}{n-1} \int_{1}^{r}\left(\frac{J^{\prime}}{J}\right)^{2}(t) d t-f^{\prime}(r) \leq(n-1) r+C_{0}
$$

for some constant $C_{0}>0$ independent of $r$. Let us denote

$$
u(t):=\frac{J_{f}^{\prime}(t)}{J_{f}(t)}=\frac{J^{\prime}}{J}(r)-f^{\prime}(r) .
$$


Then for any $r \geq 1$,

$$
u(r)+\frac{1}{n-1} \int_{1}^{r}\left(u(t)+f^{\prime}(t)\right)^{2} d t \leq(n-1) r+C_{0} .
$$

The Cauchy-Schwarz inequality implies that

$$
\int_{1}^{r}\left(u(t)+f^{\prime}(t)\right)^{2} d t \geq(r-1)^{-1}\left\{\int_{1}^{r}\left(u(t)+f^{\prime}(t)\right) d t\right\}^{2} .
$$

Therefore, from (2.1) we obtain

$$
u(r)+\frac{1}{(n-1) r}\left(f(r)-f(1)+\int_{1}^{r} u(t) d t\right)^{2} \leq(n-1) r+C_{0} .
$$

We now claim that for any $r \geq 1$,

$$
\int_{1}^{r} u(t) d t \leq(n-1+\alpha) r+\alpha+2 \beta+C_{0} .
$$

To prove this, define

$$
v(r):=(n-1+\alpha) r+\alpha+2 \beta+C_{0}-\int_{1}^{r} u(t) d t .
$$

We show instead that $v(r) \geq 0$ for all $r \geq 1$. Clearly, $v(1)>0$. Suppose that $v$ does not remain positive for all $r \geq 1$ and let $R>1$ be the first number such that $v(R)=0$. Then,

$$
\int_{1}^{R} u(t) d t=(n-1+\alpha) R+\alpha+2 \beta+C_{0}
$$

In other words,

$$
\begin{aligned}
& \frac{1}{(n-1) R}\left(f(R)-f(1)+\int_{1}^{R} u(t) d t\right)^{2} \\
& \quad=\frac{1}{(n-1) R}\left(f(R)-f(1)+(n-1+\alpha) R+\alpha+2 \beta+C_{0}\right)^{2} \\
& \quad \geq \frac{1}{(n-1) R}\left((n-1) R+C_{0}\right)^{2} \geq(n-1) R+2 C_{0} .
\end{aligned}
$$

Plugging this into (2.2), we conclude $u(R) \leq-C_{0}<0$. This shows that $v^{\prime}(R)=(n-1+\alpha)-u(R)>0$, which implies the existence of a small 
enough $\delta>0$ such that $v(R-\delta)<v(R)=0$. This obviously contradicts with the choice of $R$.

We have thus proved that (2.3) is true for any $r \geq 1$, or

$$
\log J_{f}(r)-\log J_{f}(1) \leq(n-1+\alpha) r+\alpha+2 \beta+C_{0}
$$

In particular, for $R \geq 1$, we have the volume bound of the form

$$
V_{f}\left(B_{p}(R)\right) \leq C \mathrm{e}^{(n-1+\alpha) R},
$$

with the constant $C$ depending on $\alpha, \beta$ and $B_{p}(1)$.

We remark that in the special case of $f$ being bounded, hence $\alpha=0$, the estimate becomes

$$
V_{f}\left(B_{p}(R)\right) \leq C \mathrm{e}^{(n-1) R}
$$

for $R \geq 0$. This improves a result in [28] in the sense that the rate of exponential growth for the weighted volume does not depend on $\sup _{M}|f|$.

Lemma 2.1 readily leads to the following estimate for the bottom spectrum of $\Delta_{f}$.

Theorem 2.1. Let $\left(M, g, \mathrm{e}^{-f} d v\right)$ be a complete smooth metric measure space with $\operatorname{Ric}_{f} \geq-(n-1)$. Assume the linear growth rate of $f$ is a. Then we have

$$
\lambda_{1}\left(\Delta_{f}\right) \leq \frac{1}{4}(n-1+a)^{2} .
$$

In particular, if $f$ is of sublinear growth, then the bottom spectrum of the weighted Laplacian has the following sharp upper bound:

$$
\lambda_{1}\left(\Delta_{f}\right) \leq \frac{(n-1)^{2}}{4} .
$$

Proof of Theorem 2.1. Let $\psi$ be a cut-off function on $B_{p}(R)$ such that $\psi=1$ on $B_{p}(R-1)$ and $|\nabla \psi| \leq 2$. Set $\phi(y):=\mathrm{e}^{-\frac{(n-1+a+\varepsilon)}{2} r(y)} \psi(y)$ as a test function in the variational principle for $\lambda_{1}\left(\Delta_{f}\right)$, where $\varepsilon>0$ is an arbitrary positive constant. Then, by Lemma 2.1 , we obtain

$$
\lambda_{1}\left(\Delta_{f}\right) \leq \frac{(n-1+a+\varepsilon)^{2}}{4} .
$$

Since $\varepsilon$ is arbitrary, this implies $\lambda_{1}\left(\Delta_{f}\right) \leq \frac{(n-1+a)^{2}}{4}$.

In the case that $f$ is of sublinear growth, we can take $a=0$. Therefore, $\lambda_{1}\left(\Delta_{f}\right) \leq \frac{1}{4}(n-1)^{2}$ and the theorem is proved. 


\section{3. f-harmonic functions}

In this section, we establish the following gradient estimate for positive $f$-harmonic functions defined on $M$.

Theorem 3.1. Let $\left(M^{n}, g, \mathrm{e}^{-f} d v\right)$ be a complete smooth metric measure space with $\operatorname{Ric}_{f} \geq-(n-1)$. Assume that for any $x \in M$,

$$
\sup _{y \in B_{x}(1)}|f(y)-f(x)| \leq a
$$

Then there exists a constant $C(n, a)$ depending only on $n$ and a such that for any $u>0$ with $\Delta_{f} u=0$ we have

$$
|\nabla \log u| \leq C(n, a)
$$

Under the stronger assumption that $|\nabla f| \leq a$, the result follows essentially by Yau's classical argument, see [29] for details. However, it seems no longer possible to apply Yau's approach directly once the hypothesis on $f$ only involves its oscillation on unit balls. Note that the theorem in particular is applicable to the case $f$ is bounded on $M$. Our proof of Theorem 3.1 follows the strategy in [20]. We will first obtain local Neumann Poincaré and Sobolev inequalities and then use the DeGiorgi-Nash-Moser theory. Let us first recall the following Laplace comparison theorem from [28],

$$
\Delta_{f} d(x, y) \leq(n-1) \operatorname{coth} r+\frac{2}{\sinh ^{2} r} \int_{0}^{r}(f(t)-f(r)) \cosh (2 t) d t
$$

where $r:=d(x, y), \quad f(t):=f(\gamma(t))$ and $\gamma(t)$ is a minimizing normal geodesic such that $\gamma(0)=x$ and $\gamma(r)=y$.

Using the assumption on $f$ that $|f(t)-f(r)| \leq a$, we get

$$
\Delta_{f} d(x, y) \leq(n-1+2 a) \operatorname{coth} r
$$

for any $0<r<1$. In particular, this yields

$$
\begin{aligned}
\frac{J_{f}\left(x, r_{2}, \xi\right)}{J_{f}\left(x, r_{1}, \xi\right)} \leq\left(\frac{\sinh \left(r_{2}\right)}{\sinh \left(r_{1}\right)}\right)^{n-1+2 a}, \\
\frac{J\left(x, r_{2}, \xi\right)}{J\left(x, r_{1}, \xi\right)} \leq \mathrm{e}^{2 a}\left(\frac{\sinh \left(r_{2}\right)}{\sinh \left(r_{1}\right)}\right)^{n-1+2 a},
\end{aligned}
$$

for any $0<r_{1}<r_{2}<1$. 
Now the arguments in $[2,10]$ (see also [20] for the case of smooth metric measure spaces) imply that we have the following local Neumann Poincaré and Sobolev inequalities.

Lemma 3.1. Let $\left(M, g, \mathrm{e}^{-f} d v\right)$ be a smooth metric measure space of dimension $n$ with $\operatorname{Ric}_{f} \geq-(n-1)$. Assume that for any $x \in M$,

$$
\sup _{y \in B_{x}(1)}|f(y)-f(x)| \leq a
$$

Then for $x \in M$ and $0<r<1$ we have

$$
\int_{B_{x}(r)}\left|\varphi-\varphi_{B_{x}(r)}\right|^{2} \leq C \cdot r^{2} \int_{B_{x}(r)}|\nabla \varphi|^{2}
$$

for any $\varphi \in C^{\infty}\left(B_{x}(r)\right)$, where $\varphi_{B_{x}(r)}:=V^{-1}\left(B_{x}(r)\right) \int_{B_{x}(r)} \varphi$ and the constant $C$ depending only on the dimension $n$ and $a$.

Note that the conclusion here that the constant $C$ is independent of $x$ is stronger than that in [20]. This is due to the more restrictive assumption on $f$ in Lemma 3.1 .

Lemma 3.2. Let $\left(M, g, \mathrm{e}^{-f} d v\right)$ be a smooth metric measure space of dimension $n$ with $\operatorname{Ric}_{f} \geq-(n-1)$. Assume that for any $x \in M$,

$$
\sup _{y \in B_{x}(1)}|f(y)-f(x)| \leq a
$$

Then there exist constants $\nu>2$ and $C$ depending only on $n$ and a such that

$$
\left(\int_{B_{x}(1)}\left|\varphi-\varphi_{B_{x}(1)}\right|^{\frac{2 \nu}{\nu-2}}\right)^{\frac{\nu-2}{\nu}} \leq \frac{C}{V\left(B_{x}(1)\right)^{\frac{2}{\nu}}} \int_{B_{x}(1)}|\nabla \varphi|^{2}
$$

for any $\varphi \in C^{\infty}\left(B_{x}(1)\right)$, where $\varphi_{B_{x}(1)}:=V^{-1}\left(B_{x}(1)\right) \int_{B_{x}(1)} \varphi$.

Although we have stated the Poincaré and Sobolev inequalities in Lemmas 3.1 and 3.2 in terms of the volume form $d v$, we point out that the same statements hold true with respect to $\mathrm{e}^{-f} d v$ as well with possibly a different $C$. This is because the oscillation of $f$ on $B_{x}(1)$ is assumed to be uniformly bounded.

We are now ready to prove Theorem 3.1. Our argument is a mixture of both the Bochner identity and the DeGiorgi-Nash-Moser theory (see e.g. $[14,25])$. 
Proof of Theorem 3.1. Let $u$ be a positive solution to $\Delta_{f} u=0$. Then the Bochner formula asserts that

$$
\frac{1}{2} \Delta_{f}|\nabla u|^{2}=\left|u_{i j}\right|^{2}+\left\langle\nabla \Delta_{f} u, \nabla u\right\rangle+\operatorname{Ric}_{f}(\nabla u, \nabla u) .
$$

Using the curvature lower bound, we get

$$
\Delta_{f}|\nabla u|^{2} \geq-2(n-1)|\nabla u|^{2}
$$

In view of (3.2), Lemmas 3.1 and 3.2, we may apply the Moser iteration argument (see $[14,25])$ to $|\nabla u|^{2}$ to conclude that

$$
\sup _{B_{x}\left(\frac{1}{16}\right)}|\nabla u|^{2} \leq \frac{C}{V_{f}\left(B_{x}\left(\frac{1}{8}\right)\right)} \int_{B_{x}\left(\frac{1}{8}\right)}|\nabla u|^{2} \mathrm{e}^{-f}
$$

for any $x \in M$, where $C$ depends only on $n$ and $a$.

Now let $\phi$ be a cut-off function with support in $B_{x}\left(\frac{1}{4}\right)$ such that $\phi=1$ on $B_{x}\left(\frac{1}{8}\right)$ and $|\nabla \phi| \leq 16$. Then, using $\Delta_{f} u=0$, we have

$$
\begin{aligned}
\int_{M}|\nabla u|^{2} \phi^{2} \mathrm{e}^{-f} & =-2 \int_{M} u \phi\langle\nabla u, \nabla \phi\rangle \mathrm{e}^{-f} \\
& \leq \frac{1}{2} \int_{M}|\nabla u|^{2} \phi^{2} \mathrm{e}^{-f}+2 \int_{M} u^{2}|\nabla \phi|^{2} \mathrm{e}^{-f}
\end{aligned}
$$

Therefore,

$$
\int_{M}|\nabla u|^{2} \phi^{2} \mathrm{e}^{-f} \leq 4 \int_{M} u^{2}|\nabla \phi|^{2} \mathrm{e}^{-f} .
$$

In view of (3.2), we conclude

$$
\begin{aligned}
\frac{1}{V_{f}\left(B_{x}\left(\frac{1}{8}\right)\right)} \int_{B_{x}\left(\frac{1}{8}\right)}|\nabla u|^{2} \mathrm{e}^{-f} & \leq \frac{c}{V_{f}\left(B_{x}\left(\frac{1}{8}\right)\right)} \int_{B_{x}\left(\frac{1}{4}\right)} u^{2} \mathrm{e}^{-f} \\
& \leq c \frac{V_{f}\left(B_{x}\left(\frac{1}{4}\right)\right)}{V_{f}\left(B_{x}\left(\frac{1}{8}\right)\right)}\left(\sup _{B_{x}\left(\frac{1}{4}\right)} u\right)^{2} \leq C\left(\sup _{B_{x}\left(\frac{1}{4}\right)} u\right)^{2}
\end{aligned}
$$

Combining with (3.3), we obtain

$$
|\nabla u|(x) \leq C \sup _{B_{x}\left(\frac{1}{4}\right)} u .
$$


On the other hand, using (3.2), Lemmas 3.1 and 3.2, and applying the Moser iteration argument to the equation $\Delta_{f} u=0$, we arrived at the following Harnack type inequality

$$
\sup _{B_{x}\left(\frac{1}{4}\right)} u \leq C \inf _{B_{x}\left(\frac{1}{4}\right)} u
$$

where $C$ is a constant depending only on $n$ and $a$. So we may rewrite (3.4) into

$$
|\nabla u|(x) \leq C(n, a) u(x),
$$

which is what we wanted to prove.

\section{Rigidity}

In this section, we focus on the equality case of the estimate of the bottom spectrum in Theorem 1.2 and prove the following rigidity theorem.

Theorem 4.1. Let $\left(M, g, \mathrm{e}^{-f} d v\right)$ be a complete smooth metric measure space of dimension $n \geq 3$ with $\operatorname{Ric}_{f} \geq-(n-1)$. Assume that $|\nabla f| \leq a$ on $M$ for some constant $a \geq 0$. If $\lambda_{1}\left(\Delta_{f}\right)=\frac{1}{4}(n-1+a)^{2}$, then either $M$ is connected at infinity or $f$ is constant and $M$ is a warped product $M=\mathbb{R} \times N$ with $d s_{M}^{2}=d t^{2}+h^{2}(t) d s_{N}^{2}$, where $N$ is compact. The function $h(t)=\mathrm{e}^{t}$ if $n \geq 4$ and $h(t)=\mathrm{e}^{t}$ or $h(t)=\cosh t$ if $n=3$.

Proof of Theorem 4.1. Assume that $M$ has at least two ends. We will divide our proof into two cases according to the ends being $f$-nonparabolic or $f$-parabolic. Recall that a manifold is called $f$-nonparabolic if $\Delta_{f}$ admits a positive symmetric Green's function. Otherwise, it is called $f$-parabolic. For an end of the manifold, the same definition applies, where now the Green's function refers to the one satisfying the Neumann boundary conditions.

We first deal with the case that there are at least two $f$-nonparabolic ends. Then, according to a result in [15], there is a bounded nonconstant $f$-harmonic function $u$ on $M$ such that $\int_{M}|\nabla u|^{2} \mathrm{e}^{-f}<\infty$.

By the Bochner formula, we have

$$
\Delta_{f}|\nabla u|^{2}=2\left|u_{i j}\right|^{2}+2 \operatorname{Ric}_{f}(\nabla u, \nabla u)
$$

For each $x \in M$, we may choose a local orthonormal frame $\left\{e_{1}, e_{2}, \ldots, e_{n}\right\}$ such that at $x$ we have $u_{1}=|\nabla u|$ and $u_{i}=0$ if $i>1$. Now a standard 
manipulation implies

$$
\left|u_{i j}\right|^{2} \geq\left|u_{11}\right|^{2}+2 \sum_{j=2}^{n}\left|u_{1 j}\right|^{2}+\frac{\left|\Delta u-u_{11}\right|^{2}}{n-1} .
$$

Since $\Delta_{f} u=0$, we have

$$
\begin{aligned}
\left|\Delta u-u_{11}\right|^{2} & =\left|\langle\nabla u, \nabla f\rangle-u_{11}\right|^{2} \geq\left|u_{11}\right|^{2}-2\langle\nabla u, \nabla f\rangle\left|u_{11}\right| \\
& \geq\left|u_{11}\right|^{2}-2 a|\nabla u|\left|u_{11}\right|
\end{aligned}
$$

as $|\nabla f| \leq a$. Thus,

$$
\left|u_{i j}\right|^{2} \geq \frac{n}{n-1} \sum_{j=1}^{n}\left|u_{1 j}\right|^{2}-\frac{2 a\left|u_{11}\right||\nabla u|}{n-1} .
$$

Since the orthonormal frame is chosen that $e_{1}$ is in the direction of $\nabla u$, it is easy to see

$$
|\nabla| \nabla u||^{2}=\sum_{j=1}^{n}\left|u_{1 j}\right|^{2}
$$

and

$$
\left|u_{11}\right| \leq|\nabla| \nabla u||
$$

Therefore,

$$
\left|u_{i j}\right|^{2} \geq \frac{n}{n-1}|\nabla| \nabla u||^{2}-\frac{2 a}{n-1}|\nabla| \nabla u|||\nabla u| .
$$

Together with the lower bound assumption on $\mathrm{Ric}_{f}$, we obtain

$$
|\nabla u| \Delta_{f}|\nabla u| \geq \frac{1}{n-1}|\nabla| \nabla u||^{2}-\frac{2 a|\nabla| \nabla u|||\nabla u|}{n-1}-(n-1)|\nabla u|^{2} .
$$

Now let

$$
\alpha:=\frac{n-2}{n-1}+\frac{\sqrt{n-2} a}{(n-1)^{2}} .
$$

Using the elementary inequality

$$
2|\nabla| \nabla u|||\nabla u| \leq\left.\frac{\sqrt{n-2}}{n-1}|\nabla| \nabla u\right|^{2}+\frac{n-1}{\sqrt{n-2}}|\nabla u|^{2},
$$

we can rewrite the preceding inequality into

$$
\Delta_{f}|\nabla u|^{\alpha} \geq-\left(\sqrt{n-2}+\frac{a}{n-1}\right)^{2}|\nabla u|^{\alpha} .
$$


Now the argument in [16] implies that $\lambda_{1}\left(\Delta_{f}\right) \leq\left(\sqrt{n-2}+\frac{a}{n-1}\right)^{2}$. Moreover, that the equality holds forces (4.2) into an equality also. In the case of $n \geq 4$, this contradicts with the assumption that $\lambda_{1}\left(\Delta_{f}\right)=$ $\frac{1}{4}(n-1+a)^{2}$. In the case $n=3$, this indeed becomes an equality. So (4.2) and all the inequalities used to prove (4.2) are equalities. In particular, from (4.1) we conclude $\langle\nabla f, \nabla u\rangle=0$ and $a|\nabla u|\left|u_{11}\right|=0$. Observe that we also have $\left|u_{11}\right|=|\nabla| \nabla u||$. Now if $a \neq 0$, then we conclude $|\nabla| \nabla u||=0$ and $|\nabla u|=C$ on $M$. But this contradicts with $\int_{M}|\nabla u|^{2} \mathrm{e}^{-f}<\infty$ as $\int_{M} \mathrm{e}^{-f}=\infty$ by the fact that $M$ is $f$-nonparabolic. Therefore, $a=0$ and $f$ is constant. So we are back to the standard Laplacian case. By [16], $M=\mathbb{R} \times N$ with $d s_{M}^{2}=d t^{2}+\cosh ^{2}(t) d s_{N}^{2}$, where $N$ is compact.

We now focus on the case when the manifold has exactly one $f$ nonparabolic end $E$. If $M$ admits more than one end, then the end $F:=$ $M \backslash E$ must be $f$-parabolic. Using the fact that $\lambda_{1}\left(\Delta_{f}\right)=\frac{1}{4}(n-1+a)^{2}$ and arguing as in [17], we obtain

$$
V_{f}\left(F \backslash B_{p}(R)\right) \leq C \mathrm{e}^{-(n-1+a) R} .
$$

Consider a ray $\gamma$ contained in the end $F$ and define the associated Busemann function

$$
\beta(x):=\lim _{t \rightarrow \infty}(t-d(x, \gamma(t))) .
$$

Then, on the end $F$, we have $\beta(x) \leq r(x)+c$, and on the end $E,-r(x)-c \leq$ $\beta(x) \leq-r(x)+c$ by $[18]$.

Denote by $\tau_{t}(s)$ the minimizing geodesic from $\gamma(t)$ to $x$ that is parameterized by the arc length. According to the Laplace comparison theorem in [28], we have

$$
\Delta_{f}(d(x, \gamma(t))) \leq(n-1) \operatorname{coth} r-\frac{1}{\sinh ^{2}(r)} \int_{0}^{r} f^{\prime}(s) \sinh (2 s) d s
$$

where $r:=d(x, \gamma(t))$ and $f(s):=f\left(\tau_{t}(s)\right)$. Since $|\nabla f| \leq a$, it is straightforward to see

$$
\Delta_{f}(d(x, \gamma(t))) \leq(n-1) \operatorname{coth} r+a .
$$

By the definition of the Busemann function, it is now standard to verify that the following estimate holds in the sense of distributions.

$$
\Delta_{f} \beta(x) \geq-(n-1+a)
$$


Note by the Laplacian comparison theorem that $\Delta_{f} r \leq(n-1) \operatorname{coth} r+$ $a$, we have

$$
V_{f}\left(B_{p}(R) \cap E\right) \leq C \mathrm{e}^{(n-1+a) R}
$$

for all $R>0$.

Consider the function

$$
B:=\mathrm{e}^{\frac{1}{2}(n-1+a) \beta} .
$$

Using (4.4) and the fact that $|\nabla \beta|=1$, we conclude

$$
\Delta_{f} B \geq-\frac{1}{4}(n-1+a)^{2} B
$$

Let $\phi$ be a cut-off function with support in $B_{p}(2 R)$ such that $\phi=1$ on $B_{p}(R)$ and $|\nabla \phi| \leq \frac{C}{R}$. Then,

$$
\begin{aligned}
\frac{1}{4} & (n-1+a)^{2} \int_{M}(B \phi)^{2} \mathrm{e}^{-f}+\int_{M} B\left(\Delta_{f} B\right) \phi^{2} \mathrm{e}^{-f} \\
& \leq \int_{M}|\nabla(B \phi)|^{2} \mathrm{e}^{-f}+\int_{M} B\left(\Delta_{f} B\right) \phi^{2} \mathrm{e}^{-f} \\
& =\int_{M}|\nabla \phi|^{2} B^{2} \mathrm{e}^{-f} \\
& \leq \frac{C}{R}
\end{aligned}
$$

where we have used (4.3) and (4.5) in the last inequality. Letting $R$ go to infinity and taking into account of (4.6), we conclude $\Delta_{f} B=-\frac{1}{4}(n-$ $1+a)^{2} B$. Equivalently,

$$
\Delta_{f} \beta=-(n-1+a) \text { and }|\nabla \beta|=1
$$

everywhere on $M$. So the Bochner formula implies that

$$
\begin{aligned}
0 & =\frac{1}{2} \Delta_{f}|\nabla \beta|^{2}=\left|\beta_{i j}\right|^{2}+\left\langle\nabla \Delta_{f} \beta, \nabla \beta\right\rangle+\operatorname{Ric}_{f}(\nabla \beta, \nabla \beta) \\
& \geq\left|\beta_{i j}\right|^{2}-(n-1) .
\end{aligned}
$$

On the other hand, under an orthonormal frame $\left\{e_{1}, e_{2}, \ldots, e_{n}\right\}$ so that $\beta_{1}=|\nabla \beta|=1$ and $\beta_{i}=0$ for $i>1$, one has $\beta_{11}=0$. In particular,

$$
\left|\beta_{i j}\right|^{2} \geq \frac{1}{n-1}(\Delta \beta)^{2}=\frac{1}{n-1}(n-1+a-\langle\nabla f, \nabla \beta\rangle)^{2} .
$$


Since $|\langle\nabla f, \nabla \beta\rangle| \leq a$, clearly $\left|\beta_{i j}\right|^{2} \geq n-1$. In conclusion, both (4.7) and (4.8) must be equalities. Reading from the equality case of (4.8), we assert that $\left(\beta_{i j}\right)$ is a diagonal matrix. Moreover, $\langle\nabla f, \nabla \beta\rangle=a$, which implies $\nabla f=$ $a \nabla \beta$ or $f=a \beta$ up to a constant. Using again that $\Delta_{f} \beta=-(n-1+a)$ and $\langle\nabla f, \nabla \beta\rangle=a$, we deduce that $\Delta \beta=-(n-1)$. Hence, the diagonal entries of $\left(\beta_{i j}\right)$ are given by $\beta_{11}=0$ and $\beta_{i i}=-1$ for $i \geq 2$. This information on $\left(\beta_{i j}\right)$ together with the fact that $|\nabla \beta|=1$ leads to the splitting of $M$ as a warped product $\mathbb{R} \times N$ with $d s_{M}^{2}=d t^{2}+\mathrm{e}^{-2 t} d s_{N}^{2}$. The manifold $N$ is given by the level set of the Busemann function $\beta^{-1}(0)=\{x: \beta(x)=0\}$. The splitting line is given by the integral curves of $\nabla \beta$. The manifold $N$ is necessarily compact due to the fact that $M$ is assumed to have (at least) two ends. For more details, see [17].

Now we show that in fact $f$ has to be constant in this case. Indeed, according to a standard computation of the curvature of a warped product metric, we have $\operatorname{Ric}_{i j}=\operatorname{Ric}_{i j}^{N}-(n-1) g_{i j}$ for $i, j \geq 2$, where $\operatorname{Ric}^{N}$ is the Ricci curvature of $N$. The condition that $\operatorname{Ric}_{f} \geq-(n-1)$ is then equivalent to $\operatorname{Ric}^{N} \geq a \mathrm{e}^{-2 t}$ on $N$. Since $a \geq 0$ and $t \in \mathbb{R}$ is arbitrary, this is impossible due to the compactness of $N$ unless $a=0$. This proves the theorem.

\section{Ends of expanding Ricci solitons}

In this section, we investigate the issue of whether an expanding gradient Ricci soliton is necessarily connected at infinity. Recall that an expanding gradient Ricci soliton is a Riemannian manifold $(M, g)$ such that Ric + Hess $(f)=-\frac{1}{2} g$ for some function $f$. Such $f$ is called the potential function of the soliton.

We begin by collecting some basic properties of expanding gradient Ricci solitons. First, it is known that

$$
S+|\nabla f|^{2}=-f
$$

after adding a suitable constant to $f$, where $S$ denotes the scalar curvature of $M$. Also, taking trace of the soliton equation, we obtain

$$
\Delta f+S=-\frac{n}{2}
$$

On the other hand, by the maximum principle, it was proved in $[24,32]$ that

$$
S \geq-\frac{n}{2}
$$


Moreover, if $S=-\frac{n}{2}$ at some point, then the manifold must be Einstein and the potential function $f$ is constant. Such soliton is called a trivial one.

Some elementary examples of expanding gradient Ricci solitons include $M=\mathbb{R}^{k} \times N^{n-k}$, where $N^{n-k}$ is an Einstein manifold with $\operatorname{Ric}_{N}=-\frac{1}{2} g_{N}$ and $\mathbb{R}^{k}$ the Gaussian expanding Ricci soliton with potential function $f=-\frac{1}{4}|x|^{2}$.

From (5.1) and (5.3), it is easy to see that $(-f)$ grows at most quadratically. Indeed,

$$
(-f)(x) \leq \frac{1}{4} r^{2}(x)+c r(x) .
$$

In view of this upper bound, it is natural to look for a matching lower bound for $-f$, which has been achieved in the case of shrinking gradient Ricci solitons [6]. However, in contrast to shrinking gradient Ricci solitons, in general such a pointwise lower bound is not to be expected for expanding gradient Ricci solitons. Indeed, for the preceding examples of the form $M=$ $\mathbb{R}^{n-k} \times N^{k}$, the potential function is given by $f(x, y)=-\frac{1}{4}|x|^{2}$ for $x \in \mathbb{R}^{n-k}$ and $y \in N$. If we take $N^{k}$ to be the simply connected hyperbolic space of Ricci curvature $-\frac{1}{2}$, then $N$ is noncompact and the potential function $f$ does not satisfy the desired bounds. Nonetheless, we have the following estimate concerning the potential function $f$. The result in particular implies that an expanding gradient Ricci soliton must be trivial if its potential function is of subquadratic growth. Prior to our result, it was known from [24] that if $|\nabla f|$ is bounded on $M$, then $M$ is Einstein.

Theorem 5.1. Let $(M, g, f)$ be a nontrivial complete expanding gradient Ricci soliton. Then there exists constant $C$ such that

$$
\frac{1}{4} r^{2}-C r^{\frac{3}{2}} \sqrt{\ln r} \leq \sup _{\partial B_{p}(r)}(-f)(x) \leq \frac{1}{4} r^{2}+C r
$$

for all $r>2$.

Proof of Theorem 5.1. As indicated above, we have

$$
\begin{aligned}
S+\Delta f & =-\frac{n}{2}, \\
|\nabla f|^{2}+S & =-f, \\
S & >-\frac{n}{2} .
\end{aligned}
$$

Now the upper bound readily follows from (5.4). So we only need to prove the lower bound. 
Let us denote

$$
u:=\frac{n}{2}-f=\frac{n}{2}+S+|\nabla f|^{2}>0
$$

For $k>0$, we compute

$$
\Delta \mathrm{e}^{2 k \sqrt{u}}=\left(\frac{k}{\sqrt{u}} \Delta u+\left(\frac{k^{2}}{u}-\frac{k}{2 u \sqrt{u}}\right)|\nabla u|^{2}\right) \mathrm{e}^{2 k \sqrt{u}} .
$$

From (5.4), we have

$$
\begin{aligned}
\Delta u & =\left(\frac{n}{2}+S\right) \quad \text { and } \\
|\nabla u|^{2} & =u-\left(\frac{n}{2}+S\right) .
\end{aligned}
$$

It follows that

$$
\Delta \mathrm{e}^{2 k \sqrt{u}}=k\left\{k-\frac{1}{2 \sqrt{u}}+\left(\frac{n}{2}+S\right)\left(\frac{1}{\sqrt{u}}+\frac{1}{2 u \sqrt{u}}-\frac{k}{u}\right)\right\} \mathrm{e}^{2 k \sqrt{u}} .
$$

Multiplying (5.7) by $u^{k^{2}}$ and integrating by parts on $B_{p}(r)$, we have

$$
\begin{aligned}
& \int_{B_{p}(r)} u^{k^{2}}\left(\Delta \mathrm{e}^{2 k \sqrt{u}}\right) \\
& =-\int_{B_{p}(r)}\left\langle\nabla \mathrm{e}^{2 k \sqrt{u}}, \nabla u^{k^{2}}\right\rangle+\int_{\partial B_{p}(r)} u^{k^{2}} \frac{\partial}{\partial r}\left(\mathrm{e}^{2 k \sqrt{u}}\right) \\
& \leq-k^{3} \int_{B_{p}(r)}|\nabla u|^{2} u^{k^{2}-\frac{3}{2}} \mathrm{e}^{2 k \sqrt{u}}+k \int_{\partial B_{p}(r)} \frac{1}{\sqrt{u}} u^{k^{2}}|\nabla u| \mathrm{e}^{2 k \sqrt{u}} \\
& =-k^{3} \int_{B_{p}(r)} \frac{1}{\sqrt{u}} u^{k^{2}} \mathrm{e}^{2 k \sqrt{u}}+k^{3} \int_{B_{p}(r)}\left(\frac{n}{2}+S\right) \frac{1}{u \sqrt{u}} u^{k^{2}} \mathrm{e}^{2 k \sqrt{u}} \\
& \quad+k \int_{\partial B_{p}(r)} \frac{1}{\sqrt{u}} u^{k^{2}}|\nabla u| \mathrm{e}^{2 k \sqrt{u}}
\end{aligned}
$$

where in the last line we have used (5.6). Consequently, from (5.7) it follows:

$$
\begin{aligned}
& \int_{\partial B_{p}(r)} \frac{|\nabla u|}{\sqrt{u}} u^{k^{2}} \mathrm{e}^{2 k \sqrt{u}} \\
& \geq \int_{B_{p}(r)}\left\{k+\frac{1}{\sqrt{u}}\left(k^{2}-\frac{1}{2}\right)+\left(\frac{n}{2}+S\right)\left(\frac{1}{\sqrt{u}}-\frac{1}{u \sqrt{u}}\left(k^{2}-\frac{1}{2}\right)-\frac{k}{u}\right)\right\} \\
& \quad \times u^{k^{2}} \mathrm{e}^{2 k \sqrt{u}} .
\end{aligned}
$$


We now claim that

$$
k+\frac{1}{\sqrt{u}}\left(k^{2}-\frac{1}{2}\right)+\left(\frac{n}{2}+S\right)\left(\frac{1}{\sqrt{u}}-\frac{1}{u \sqrt{u}}\left(k^{2}-\frac{1}{2}\right)-\frac{k}{u}\right) \geq k \frac{|\nabla u|}{\sqrt{u}} .
$$

We prove this directly by checking it at arbitrary point $x \in M$. Let us denote for simplicity

$$
\alpha:=\frac{n}{2}+S(x),
$$

and let

$$
\gamma:=\sqrt{\frac{u(x)}{\alpha}} \geq 1
$$

The fact that $\gamma \geq 1$ follows from (5.6). Notice that (5.9) is equivalent to

$$
k \sqrt{u}+\left(k^{2}-\frac{1}{2}\right)+\alpha\left(1-\frac{1}{u}\left(k^{2}-\frac{1}{2}\right)-\frac{k}{\sqrt{u}}\right) \geq k \sqrt{u-\alpha} .
$$

This inequality is rewritten into the following equivalent form after replacing $u$ in terms of $\gamma$ and rearranging the terms.

$$
\alpha-k \sqrt{\alpha}\left(\frac{1}{\gamma}-\gamma+\sqrt{\gamma^{2}-1}\right)+\left(k^{2}-\frac{1}{2}\right)\left(1-\frac{1}{\gamma^{2}}\right) \geq 0 .
$$

The discriminant of this quadratic inequality in $\sqrt{\alpha}$ is given by

$$
\begin{aligned}
D & :=k^{2}\left(\frac{1}{\gamma}-\gamma+\sqrt{\gamma^{2}-1}\right)^{2}-4\left(k^{2}-\frac{1}{2}\right)\left(1-\frac{1}{\gamma^{2}}\right) \\
& =\left(1-\frac{1}{\gamma^{2}}\right)\left\{k^{2}\left(\gamma-\sqrt{\gamma^{2}-1}\right)^{2}-4\left(k^{2}-\frac{1}{2}\right)\right\}
\end{aligned}
$$

Since

$$
0 \leq \gamma-\sqrt{\gamma^{2}-1} \leq 1 \text { and } \gamma \geq 1
$$

it follows that for $k \geq 1$,

$$
D \leq\left(1-\frac{1}{\gamma^{2}}\right)\left\{-3 k^{2}+2\right\} \leq 0 .
$$

This proves that (5.10) is true for any $\gamma \geq 1$ and for any $\alpha \geq 0$. Therefore, (5.9) holds true at any $x \in M$. 
From (5.8) and (5.9) we get that

$$
k \int_{B_{p}(r)}|\nabla u| u^{k^{2}-\frac{1}{2}} \mathrm{e}^{2 k \sqrt{u}} \leq \int_{\partial B_{p}(r)}|\nabla u| u^{k^{2}-\frac{1}{2}} \mathrm{e}^{2 k \sqrt{u}}
$$

Hence the function

$$
w(r):=\int_{B_{p}(r)}|\nabla u| u^{k^{2}-\frac{1}{2}} \mathrm{e}^{2 k \sqrt{u}}
$$

satisfies $k w(r) \leq w^{\prime}(r)$ for any $r \geq 0$. Since $(M, g, f)$ is assumed to be a nontrivial Ricci soliton, there exists a positive radius $r_{0}$ for which $w\left(r_{0}\right)>0$. Integrating $k w(t) \leq w^{\prime}(t)$ from $t=r_{0}$ to $t=r$, we conclude that there exists a positive constant $C>0$ such that $w(r) \geq C \mathrm{e}^{k r}$. Therefore, we have proved that

$$
\int_{\partial B_{p}(r)}|\nabla u| u^{k^{2}-\frac{1}{2}} \mathrm{e}^{2 k \sqrt{u}} \geq C \mathrm{e}^{k r}, \quad \text { for } r \geq r_{0}
$$

We now prove that there exists a constant $c(n)$ depending only on $n$ such that

$$
A\left(\partial B_{p}(r)\right) \leq C \mathrm{e}^{c(n) r}, \quad \text { for } r \geq r_{0}
$$

Let us stress that (5.12) refers to the usual area, not the weighted one. This claim follows as in Lemma 2.1. Indeed, from Lemma 2.1 we have

$$
\frac{J^{\prime}}{J}(r)+\frac{1}{n-1} \int_{1}^{r}\left(\frac{J^{\prime}}{J}\right)^{2}(t) d t \leq f^{\prime}(r)+\frac{1}{2} r+C_{0} .
$$

Since

$$
\sup _{B_{p}(r)}|\nabla f| \leq \frac{1}{2} r+c
$$

it follows that

$$
\frac{J^{\prime}}{J}(r)+\frac{1}{(n-1) r}\left(\int_{1}^{r} \frac{J^{\prime}}{J}(t) d t\right)^{2} \leq r+C_{0} .
$$

The argument in Lemma 2.1 now shows that

$$
A\left(\partial B_{p}(r)\right) \leq C \mathrm{e}^{2 \sqrt{n-1} r} .
$$


This proves (5.12) is true. Plugging this into (5.11) and using that

$$
|\nabla u|^{2} \leq u \leq \frac{1}{4} r^{2}+c r
$$

we have

$$
\sup _{\partial B_{p}(r)} \mathrm{e}^{2 k \sqrt{u}} \geq C \mathrm{e}^{k r-2 k^{2} \ln r-c(n) r}
$$

In other words,

$$
\sup _{\partial B_{p}(r)} 2 \sqrt{u} \geq r-2 k \ln r-c \frac{r}{k} .
$$

Since this estimate is true for each fixed $r$ over all $k$, we may optimize by choosing $k=\sqrt{\frac{r}{\ln r}}$. It is easy to see that this proves the Theorem.

Theorem 5.1 shows that the results we proved in Section 4 cannot be applied directly to expanding gradient Ricci solitons as the boundedness assumption on $|\nabla f|$ is not available. To address the issue of connectedness at infinity, we have to proceed somewhat differently to obtain a $\lambda_{1}$ estimate. Also, our proof to rule out the existence of small ends seems to rely on some specific properties of expanding gradient Ricci solitons.

Theorem 5.2. Let $(M, g, f)$ be a complete gradient expanding Ricci soliton. Assume that $S \geq-\frac{n-1}{2}$ on $M$. Then either $M$ is connected at infinity or $M=\mathbb{R} \times N^{n-1}$, where $N$ is a compact Einstein manifold and $\mathbb{R}$ is the Gaussian expanding soliton.

Before proving the theorem, we first establish a weighted Poincaré inequality for expanding gradient Ricci solitons. The importance of a weighted Poincaré inequality for the issue of connectedness at infinity of Riemannian manifolds has been exemplified in [19].

Lemma 5.1. Let $(M, g, f)$ be a complete nontrivial expanding gradient Ricci soliton. Define $\sigma:=S+\frac{n}{2}$. Then $\sigma>0$ on $M$ and

$$
\int_{M} \sigma \phi^{2} \mathrm{e}^{-f} \leq \int_{M}|\nabla \phi|^{2} \mathrm{e}^{-f}
$$

for any $\phi \in C_{0}^{\infty}(M)$. 
Proof of Lemma 5.1. The fact that $\sigma>0$ is clear from (5.3). Using (5.1) and (5.2), we compute

$$
\Delta_{f} \mathrm{e}^{f}=\left(\Delta_{f}(f)+|\nabla f|^{2}\right) \mathrm{e}^{f}=(\Delta f) \mathrm{e}^{f}=-\left(\frac{n}{2}+S\right) \mathrm{e}^{f} .
$$

Now Proposition 1.1 in [19] implies the claimed weighted Poincaré inequality.

Using Lemma 5.1, we proceed to prove the theorem.

Proof of Theorem 5.2. Since $M$ satisfies a weighted Poincaré inequality, from [19] it follows that $M$ is $f$-nonparabolic. By hypothesis $S \geq-\frac{n-1}{2}$, we see that

$$
\sigma=\frac{n}{2}+S \geq \frac{1}{2}
$$

i.e., the bottom spectrum of the weighted Laplacian on $M$ satisfies $\lambda_{1}\left(\Delta_{f}\right) \geq \frac{1}{2}$.

We first show that all ends of $M$ must be $f$-nonparabolic. Suppose $E$ is an $f$-parabolic end of $M$. Let us observe first that both $|f|$ and $|\nabla f|$ must be bounded on $E$. To see this, let us denote by

$$
u:=n-2 f
$$

It is easy to check, using (5.4), that $u$ has the following properties on $M$ :

$$
\begin{aligned}
u & \geq 1, \\
\Delta_{f} u & =u, \\
|\nabla u|^{2} & \leq 2 u .
\end{aligned}
$$

Consequently, a direct computation shows that the function $w:=\mathrm{e}^{-\frac{1}{2} u}>0$ verifies $\Delta_{f} w \leq 0$. If $u$ is an unbounded function on $E$, then $w$ is a positive $f$-superharmonic function on $E$, which achieves its infimum at the infinity of $E$. It is well known that this implies that $E$ is $f$-nonparabolic, see [13] for details. This contradicts our assumption that $E$ is $f$-parabolic, therefore $u$ must be bounded on $E$. In particular, there exists a constant $A>0$ such that

$$
|f|+|\nabla f| \leq A \quad \text { on } E .
$$

By the Laplace comparison theorem in [28], or cf. (3.1) here, it follows that for any two points $x, y \in E$,

$$
\Delta_{f} d(x, y) \leq a \operatorname{coth} d(x, y)
$$


for some constant $a$ independent of $x$ or $y$. It is standard to obtain from here that

$$
V_{f}\left(B_{x}(1)\right) \geq C_{1} \mathrm{e}^{-C_{2} r(x)}
$$

for $C_{1}$ and $C_{2}$ independent of $x$. Here, $r(x):=d(p, x)$, for $p \in M$ fixed.

In the following, we obtain a contradiction to (5.14). Since $\sigma \geq \frac{1}{2}$, it follows by [16] that

$$
\int_{E \backslash E(r)} \mathrm{e}^{-f} \leq C \mathrm{e}^{-\sqrt{2} r}
$$

where $E(r):=E \cap B_{p}(r)$ and $C$ is a constant independent of $r$. Since $\Delta_{f} u=u$, it is easy to see that

$$
\Delta_{f} u^{k} \geq k u^{k}, \quad \text { for all } k \geq 1 \text {. }
$$

We claim that Li-Wang's result in [16] implies

$$
\int_{E \backslash E(r)} u^{2 k} \mathrm{e}^{-f} \leq \mathrm{e}^{-\sqrt{4 k+2}\left(r-r_{0}\right)} \int_{E\left(r_{0}\right) \backslash E\left(r_{0}-1\right)} u^{2 k} \mathrm{e}^{-f},
$$

for any $r>2 r_{0}$ and any positive integer $k$. For that, we need only to check that

$$
\frac{1}{R} \int_{E(R)} u^{2 k} \mathrm{e}^{-\sqrt{4 k+2} r} \mathrm{e}^{-f} \rightarrow 0 \quad \text { as } R \rightarrow \infty .
$$

This is clearly implied by the fact that $u$ is bounded on $E$ and by (5.15). Hence, the claim (5.16) is true. Since $u \geq 1$ is bounded, we see from (5.16) that for any $k \geq 1$, there exists a constant $C(k)$ so that

$$
\int_{E \backslash E(r)} \mathrm{e}^{-f} \leq C(k) \mathrm{e}^{-\sqrt{4 k+2} r}, \quad \text { for all } r>2 r_{0} .
$$

Choosing $k$ large enough, this clearly contradicts with (5.14). In conclusion, all ends of $M$ must be $f$-nonparabolic.

We now deal with the $f$-nonparabolic ends. Suppose $M$ has at least two ends. Let $E$ be an $f$-nonparabolic end. Then it follows that $F:=M \backslash E$ is also an $f$-nonparabolic end. By [15], there exists an $f$-harmonic function $h$ on $M$ such that $0<h<1$ and $\int_{M}|\nabla h|^{2} \mathrm{e}^{-f}<\infty$. 
Now the Bochner formula together with the Kato inequality shows

$$
\frac{1}{2} \Delta_{f}|\nabla h|^{2}=\left|h_{i j}\right|^{2}-\frac{1}{2}|\nabla h|^{2} \geq\left.|\nabla| \nabla h\right|^{2}-\frac{1}{2}|\nabla h|^{2}
$$

or

$$
\Delta_{f}|\nabla h| \geq-\frac{1}{2}|\nabla h| .
$$

We use this in the weighted Poincaré inequality to find that for any cut-off function $\phi$,

$$
\begin{aligned}
\int_{M} & \sigma|\nabla h|^{2} \phi^{2} \mathrm{e}^{-f} \leq \int_{M}|\nabla(|\nabla h| \phi)|^{2} \mathrm{e}^{-f} \\
& =\left.\left.\int_{M}|\nabla| \nabla h\right|^{2}\left|\phi^{2} \mathrm{e}^{-f}+\frac{1}{2} \int_{M}\left\langle\nabla|\nabla h|^{2}, \nabla \phi^{2}\right\rangle \mathrm{e}^{-f}+\int_{M}\right| \nabla \phi\right|^{2}|\nabla h|^{2} \mathrm{e}^{-f} \\
& =-\int_{M}|\nabla h|\left(\Delta_{f}|\nabla h|\right) \phi^{2} \mathrm{e}^{-f}+\int_{M}|\nabla \phi|^{2}|\nabla h|^{2} \mathrm{e}^{-f} \\
& \leq \frac{1}{2} \int_{M}|\nabla h|^{2} \phi^{2} \mathrm{e}^{-f}+\int_{M}|\nabla \phi|^{2}|\nabla h|^{2} \mathrm{e}^{-f}
\end{aligned}
$$

Notice that we may choose $\phi$ so that

$$
\int_{M}|\nabla \phi|^{2}|\nabla h|^{2} \mathrm{e}^{-f} \rightarrow 0 .
$$

Since $\sigma \geq \frac{1}{2}$, this implies $\sigma=\frac{1}{2}$ or $S=-\frac{n}{2}+\frac{1}{2}$ on $M$. The rigidity of gradient Ricci solitons with constant scalar curvature has been studied in [22]. Our situation here is more special since we have a specific value for $S$. Let us denote

$$
v:=2 \sqrt{-f+\frac{n-1}{2}}=2|\nabla f| .
$$

Notice that $|\nabla v|=1$ at points where $v \neq 0$. Moreover, observe that since

$$
\begin{aligned}
& \Delta_{f} v^{2}=4 \Delta_{f}(-f)=4\left(\frac{n}{2}-f\right)=v^{2}+2 \\
& \Delta_{f} v^{2}=2 v \Delta_{f} v+2|\nabla v|^{2}=2 v \Delta_{f} v+2
\end{aligned}
$$

we get that $\Delta_{f} v=\frac{1}{2} v$ whenever $v \neq 0$. This implies that $v$ is in fact smooth and $|\nabla v|=1$ everywhere on $M$. The Bochner formula gives

$$
0=\frac{1}{2} \Delta_{f}|\nabla v|^{2}=\left|v_{i j}\right|^{2}-\frac{1}{2}|\nabla v|^{2}+\left\langle\nabla \Delta_{f} v, \nabla v\right\rangle=\left|v_{i j}\right|^{2}
$$

Therefore, $v_{i j}=0$ and $M$ admits a parallel vector field $\nabla v$. So $M$ is isometric to $\mathbb{R} \times N$. Since $M$ is assumed to have two ends, $N$ must be a compact 
expanding gradient Ricci soliton. But it is well known that $N$ then has to be Einstein. The Theorem is proved.

\section{Volume of shrinking Ricci solitons}

Consider a shrinking gradient Ricci soliton $(M, g, f)$. By definition, this is a Riemannian manifold $(M, g)$ for which there exists a smooth $f$ so that Ric + Hess $(f)=\frac{1}{2} g$. Taking the trace of this equation, we also obtain $S+$ $\Delta f=\frac{n}{2}$, where $S$ denotes the scalar curvature of $M$. It is well known [11] that $S+|\nabla f|^{2}=f$ after adjusting $f$ by adding a suitable constant. With this normalization of $f$ the Perelman's invariant is defined by $[7,21]$ :

$$
\mu_{0}:=-\log \left((4 \pi)^{-\frac{n}{2}} \int_{M} \mathrm{e}^{-f}\right)<\infty .
$$

In this section we prove that the volume of a complete noncompact, shrinking gradient Ricci soliton is of at least linear growth. As pointed out in the introduction, this result is optimal. The corresponding result for manifolds with nonnegative Ricci curvature was proved independently by Calabi [4] and Yau [31].

Theorem 6.1. Let $(M, g, f)$ be a complete noncompact, shrinking gradient Ricci soliton. Then there exists a constant $C>0$ depending only on dimension $n$ and the Perelman's invariant $\mu_{0}$ defined above such that

$$
\operatorname{Vol}\left(B_{p}(r)\right) \geq C r
$$

for all $r \geq r_{0}$, where $p$ is a minimum point of $f$ and $r_{0}$ depends only on $n$.

Our proof of Theorem 6.1 involves Perelman's ideas in [21, 26] and a logarithmic Sobolev inequality for shrinking gradient Ricci solitons [7]. The main difference here is that no extra assumptions are imposed on the curvature.

The proof of the Theorem consists of several steps. We begin by proving that the volume of unit balls decay at most exponentially on a noncompact shrinking gradient Ricci soliton. This may be of independent interest. We then show that the volume of any noncompact shrinking gradient Ricci soliton must be infinite in Lemma 6.2, a fact which also appeared in [5]. However, our approach here is different. With this fact, we then complete our argument for Theorem 6.1. 
Lemma 6.1. Let $(M, g, f)$ be a complete noncompact, shrinking gradient Ricci soliton. Then there exists a constant $C(n)>0$ depending only on dimension $n$ such that

$$
V\left(B_{x}(1)\right) \geq V\left(B_{p}(1)\right) \mathrm{e}^{-C(n) d(p, x)}
$$

for all $x \in M$, where $p$ is a minimum point of $f$.

Proof of Lemma 6.1. Take any point $x \in M$ and express the volume form in the geodesic polar coordinates centered at $x$ as

$$
\left.d V\right|_{\exp _{x}(r \xi)}=J(x, r, \xi) d r d \xi
$$

for $r>0$ and $\xi \in S_{x} M$, a unit tangent vector at $x$. In the following, we will omit the dependence of these quantities on $\xi$. Let $x \in M$ be arbitrary and

$$
r:=d(p, x)
$$

Notice that by the triangle inequality, $B_{p}(1) \subset B_{x}(r+1) \backslash B_{x}(r-1)$. Let $\gamma(s)$ be a minimizing geodesic starting from $x$, such that $\gamma(0)=x$ and $\gamma(T) \in B_{p}(1)$, for some

$$
r-1 \leq T \leq r+1
$$

Along $\gamma$ we have, by a standard formula:

$$
\frac{J^{\prime}}{J}(t) \leq \frac{n-1}{t}-\frac{1}{t^{2}} \int_{0}^{t} s^{2} \operatorname{Ric}\left(\gamma^{\prime}(s), \gamma^{\prime}(s)\right) d s .
$$

Using the soliton equation that

$$
\operatorname{Ric}\left(\gamma^{\prime}(s), \gamma^{\prime}(s)\right)=\frac{1}{2}-\operatorname{Hess}(f)\left(\gamma^{\prime}(s), \gamma^{\prime}(s)\right)
$$

and then integrating by parts we obtain:

$$
\frac{J^{\prime}}{J}(t) \leq \frac{n-1}{t}-\frac{1}{6} t+f^{\prime}(t)-\frac{2}{t^{2}} \int_{0}^{t} s f^{\prime}(s) d s,
$$

where $f(s):=f(\gamma(s))$ and $0 \leq t \leq T$. We note that by the triangle inequality

$$
d(p, \gamma(s)) \leq d(p, \gamma(T))+d(\gamma(T), \gamma(s)) \leq T-s+1
$$


and

$$
d(p, \gamma(s)) \geq d(\gamma(T), \gamma(s))-d(p, \gamma(T)) \geq T-s-1
$$

So by the estimates of $f$ proved in $[6,12]$ together with $(6.1)$ we obtain that

$$
\begin{aligned}
\frac{1}{4}\left[(r-s-c(n))_{+}\right]^{2} & \leq f(\gamma(s)) \leq \frac{1}{4}(r-s+c(n))^{2} \quad \text { and } \\
|\nabla f|(\gamma(s)) & \leq \frac{1}{2}(r-s+c(n)),
\end{aligned}
$$

where $c(n)$ is a constant depending only on $n$ and $a_{+}:=\max \{a, 0\}$. In the following, we will denote by $c$ a constant depending only on $n$, which may change from line to line.

Plugging this into (6.2) results in

$$
\begin{aligned}
\frac{J^{\prime}}{J}(t) & \leq \frac{n-1}{t}-\frac{1}{6} t+f^{\prime}(t)+\frac{1}{t^{2}} \int_{0}^{t} s(r-s+c) d s \\
& =\frac{n-1}{t}+c+f^{\prime}(t)+\frac{1}{2}(r-t) .
\end{aligned}
$$

Integrating from $t=1$ to $t=T$ we obtain that

$$
\log \frac{J(T)}{J(1)} \leq c T+f(T)-f(1)+\frac{1}{2}\left(r T-\frac{1}{2} T^{2}\right) .
$$

Notice that $f(T) \leq c$ as $\gamma(T) \in B_{p}(1)$. On the other hand, by (6.3),

$$
f(1)=f(\gamma(1)) \geq \frac{1}{4} r^{2}-c(n) r .
$$

Hence, combining with (6.1), one sees from (6.4) that

$$
\log \frac{J(T)}{J(1)} \leq c r
$$

In other words,

$$
J(x, T, \xi) \leq \mathrm{e}^{c d(p, x)} J(x, 1, \xi), \quad \text { whenever } \exp _{x}(T \xi) \in B_{p}(1) .
$$

By integrating this over a subset of $S_{x} M$ consisting of all unit tangent vectors $\xi$ so that $\exp _{x}(T \xi) \in B_{p}(1)$ for some $T$, it follows that

$$
V\left(B_{p}(1)\right) \leq \mathrm{e}^{c d(p, x)} A\left(\partial B_{x}(1)\right),
$$

for a constant $c$ depending only on $n$. 
The same argument implies in fact that

$$
V\left(B_{p}(1)\right) \leq \mathrm{e}^{c d(p, x)} A\left(\partial B_{x}(\rho)\right),
$$

for all $1 / 2 \leq \rho \leq 1$. After integrating with respect to $\rho$, we have

$$
V\left(B_{x}(1)\right) \geq V\left(B_{p}(1)\right) \mathrm{e}^{-c d(p, x)} .
$$

The lemma is proved.

We now establish the fact that the volume of a noncompact shrinking gradient Ricci soliton is infinite.

Lemma 6.2. Let $(M, g, f)$ be a complete noncompact, shrinking gradient Ricci soliton. Then $\operatorname{Vol}(M)=\infty$.

Proof of Lemma 6.2. Suppose to the contrary that

$$
\operatorname{Vol}(M)=V<\infty
$$

Define

$$
\rho:=2 \sqrt{f} \text { and } D(t):=\{\rho \leq t\} .
$$

According to $[6,12]$,

$$
d(p, x)-a \leq \rho(x) \leq d(p, x)+a,
$$

for a constant $a>0$ depending only on $n$. Moreover, it is easy to see that $|\nabla \rho| \leq 1$ on $M$. We let

$$
V(t):=\operatorname{Vol}(D(t)) \quad \text { and } \quad \chi(t):=\int_{D(t)} S
$$

By the co-area formula, we have

$$
V^{\prime}(t)=\int_{\partial D(t)} \frac{1}{|\nabla \rho|} \quad \text { and } \quad \chi^{\prime}(t)=\int_{\partial D(t)} \frac{S}{|\nabla \rho|} .
$$

Let us now recall the logarithmic Sobolev inequality from [7], which holds for any compactly supported Lipschitz function $u$ on $M$.

$$
\int_{M} u^{2} \log u^{2}-\left(\int_{M} u^{2}\right) \log \left(\int_{M} u^{2}\right) \leq \mu_{0} \int_{M} u^{2}+4 \int_{M}|\nabla u|^{2}+\int_{M} S u^{2} .
$$


For $t \geq 2$, we define $u_{t}: \mathbb{R} \rightarrow \mathbb{R}$ by

$$
u_{t}(s)= \begin{cases}1, & \text { on } s \geq t \\ s-(t-1), & \text { on } t-1 \leq s \leq t \\ 0 & \text { on } 0 \leq s \leq t-1\end{cases}
$$

and a function on $M$ by $u_{t}(x):=u_{t}(\rho(x))$.

Using the fact that $S \geq 0$ (see $[5,8]$ ) and $\chi(\infty)=\int_{M} S \leq \frac{n}{2} \operatorname{Vol}(M)<$ $\infty$ (see [6]), one easily justifies that such $u_{t}$ is admissible in the preceding logarithmic Sobolev inequality (6.5). Let us denote

$$
y(t):=\int_{M} u_{t}^{2}
$$

The log-Sobolev inequality applied to $u_{t}$ implies

$$
-y(t) \log y(t) \leq C(\operatorname{Vol}(M)-V(t-1))+\int_{M} S u_{t}^{2},
$$

where $C$ depends only on $n$ and the Perelman's invariant $\mu_{0}$. We have also used above the elementary inequality $u_{t}^{2} \log u_{t}^{2} \geq-\frac{1}{e}$.

Since $y(t) \geq \operatorname{Vol}(M)-V(t)$, we obtain

$$
-y(t) \log y(t) \leq C y(t-1)+\int_{M} S u_{t}^{2} .
$$

We now wish to express the term $\int_{M} S u_{t}^{2}$ from (6.7) in terms of $y(t)$. For any $T>t$, since $S=\frac{n}{2}-\Delta f$, we have

$$
\begin{aligned}
\int_{D(T)} S u_{t}^{2} & =\frac{n}{2} \int_{D(T)} u_{t}^{2}-\int_{D(T)}(\Delta f) \cdot u_{t}^{2} \\
& =\frac{n}{2} \int_{D(T)} u_{t}^{2}+\int_{D(T)}\left\langle\nabla f, \nabla u_{t}^{2}\right\rangle-\int_{\partial D(T)} \frac{\partial f}{\partial \nu} u_{t}^{2},
\end{aligned}
$$

where $\nu$ is the unit normal to $\partial D(t)$. In fact, since $\frac{\partial}{\partial \nu}=\frac{\nabla \rho}{|\nabla \rho|}$, it follows that $\frac{\partial f}{\partial \nu}=\frac{1}{2} \rho|\nabla \rho| \geq 0$. Moreover, observe that $\left\langle\nabla f, \nabla u_{t}^{2}\right\rangle$ has support in $D(t) \backslash D(t-1)$ and in that region we have

$$
\begin{aligned}
\left\langle\nabla f, \nabla u_{t}^{2}\right\rangle & =2\langle\nabla f, \nabla \rho\rangle u_{t}=\rho|\nabla \rho|^{2} u_{t} \\
& \leq \rho u_{t} .
\end{aligned}
$$


Using this in (6.8) we find that

$$
\int_{D(T)} S u_{t}^{2} \leq \frac{n}{2} \int_{D(T)} u_{t}^{2}+\int_{D(t) \backslash D(t-1)} \rho u_{t} .
$$

Hence, we can let $T \rightarrow \infty$ in the above and get

$$
\int_{M} S u_{t}^{2} \leq \frac{n}{2} y(t)+t \int_{D(t) \backslash D(t-1)} u_{t} .
$$

By a direct calculation it follows that

$$
\frac{d}{d t} y(t)=\frac{d}{d t} \int_{M} u_{t}^{2}=-2 \int_{D(t) \backslash D(t-1)} u_{t} .
$$

Therefore, combining this with (6.9) we get

$$
\int_{M} S u_{t}^{2} \leq \frac{n}{2} y(t)-\frac{1}{2} t y^{\prime}(t) .
$$

We use this in (6.7) to conclude that

$$
t y^{\prime}(t)-2 y(t) \log y(t) \leq C y(t-1)
$$

for a constant $C$ depending only on $n$ and the Perelman's invariant $\mu_{0}$. This inequality is true for $t \geq c(n)$, where $c(n)$ is a large enough constant so that $D(t)$ are nonempty.

In the following, we will use this differential inequality to show that the function $y(t)$ decays exponentially with arbitrarily large exponent. Here, our argument is inspired by [23]. We first show $y(t)$ is of exponential decay of some order. We let

$$
\delta:=\mathrm{e}^{-C}>0
$$

where $C$ is the constant from (6.11). There exists $\varepsilon>0$ sufficiently small so that

$$
y\left(\frac{1}{\varepsilon}\right)<\delta \mathrm{e}^{-2} .
$$

Indeed, this is because $\operatorname{Vol}(M)<\infty$, hence

$$
\lim _{t \rightarrow \infty} y(t)=0
$$

Clearly, we can assume $\mathrm{e}^{\varepsilon}<2$. Let us define $t_{0}:=\frac{1}{\varepsilon}$. We claim that

$$
y(t)<\delta \mathrm{e}^{-\varepsilon t}, \quad \text { for any } t_{0} \leq t \leq t_{0}+1
$$


Indeed, by (6.10) we know that $y(t)$ is decreasing in $t$, therefore

$$
y(t) \leq y\left(t_{0}\right)=y\left(\frac{1}{\varepsilon}\right)<\delta \mathrm{e}^{-2},
$$

for any $t_{0} \leq t \leq t_{0}+1$. However, since $t_{0} \varepsilon=1$, we can write

$$
\delta \mathrm{e}^{-2}=\delta \mathrm{e}^{-2 \varepsilon t_{0}}<\delta \mathrm{e}^{-\varepsilon t}
$$

where the last inequality is true because $t \leq t_{0}+1<2 t_{0}$. Hence, this proves that (6.12) is true.

Now we claim that

$$
y(t)<\delta \mathrm{e}^{-\varepsilon t}
$$

for any $t_{0} \leq t$. If (6.13) fails to be true for all $t \geq t_{0}$, there exists a first $t=r$ so that $y(r)=\delta \mathrm{e}^{-\varepsilon r}$. Then the choice of $r$ implies

$$
\begin{gathered}
y(r)=\delta \mathrm{e}^{-\varepsilon r}, \\
y^{\prime}(r) \geq-\varepsilon \delta \mathrm{e}^{-\varepsilon r} .
\end{gathered}
$$

Since (6.13) is true for $t \leq t_{0}+1$, we know that $r-1 \geq t_{0}$. Consequently, $y(r-1) \leq \delta \mathrm{e}^{-\varepsilon(r-1)}$. Now (6.11) for $t=r$ implies that

$$
\begin{aligned}
-\varepsilon \delta r \mathrm{e}^{-\varepsilon r}+2 \delta \mathrm{e}^{-\varepsilon r}(-\log \delta+\varepsilon r) & \leq r y^{\prime}(r)-2 y(r) \log y(r) \\
& \leq C y(t-1) \\
& \leq C \delta \mathrm{e}^{\varepsilon} \mathrm{e}^{-\varepsilon r}
\end{aligned}
$$

Simplifying this gives

$$
\varepsilon r-2 \log \delta \leq C \mathrm{e}^{\varepsilon} .
$$

However, $\varepsilon r \geq \varepsilon t_{0}=1$ and $\mathrm{e}^{\varepsilon}<2$ from the choice of $\varepsilon$. Since by definition $\delta:=\mathrm{e}^{-C}$, this is a contradiction.

Hence (6.13) is true for all $t \geq t_{0}$. In particular,

$$
y(t) \leq \mathrm{e}^{-\varepsilon t}, \quad \text { for all } t \geq t_{0}
$$

We have thus shown that $y(t)$ decays exponentially. We now show that $y(t)$ has arbitrarily large exponential decay rate. 
Let us prove by induction on $m$ that there exists $t_{m}$ such that

$$
y(t) \leq \mathrm{e}^{-\left(\frac{3}{2}\right)^{m} \varepsilon t}, \quad \text { for all } t \geq t_{m} .
$$

Clearly, (6.14) means that (6.15) is true for $m=0$. We now assume that (6.15) is true for $m \geq 0$ and prove so for $m+1$.

For a constant $t_{m+1} \geq t_{m}$ to be picked later, let $A$ be a constant so that

$$
y(t)<A \mathrm{e}^{-\frac{7}{4}\left(\frac{3}{2}\right)^{m} \varepsilon t}, \quad \text { for all } t_{m+1} \leq t \leq t_{m+1}+1 .
$$

If this inequality holds true for all $t \geq t_{m+1}$, then by possibly renaming $t_{m+1}$ to be a larger number, (6.15) holds for $m+1$ and the induction is complete. Otherwise, there exists the first $r>t_{m+1}+1$ so that $y(r)=A \mathrm{e}^{-\frac{7}{4}\left(\frac{3}{2}\right)^{m} \varepsilon r}$. Then,

$$
\begin{aligned}
y(r) & =A \mathrm{e}^{-\frac{7}{4}\left(\frac{3}{2}\right)^{m} \varepsilon r}, \\
y^{\prime}(r) & \geq-\frac{7}{4}\left(\frac{3}{2}\right)^{m} A \varepsilon \mathrm{e}^{-\frac{7}{4}\left(\frac{3}{2}\right)^{m} \varepsilon r} .
\end{aligned}
$$

Therefore, by (6.11) we get

$$
-\frac{7}{4}\left(\frac{3}{2}\right)^{m} A \varepsilon \mathrm{e}^{-\frac{7}{4}\left(\frac{3}{2}\right)^{m} \varepsilon r} \leq 2 \frac{1}{r} y(r) \log y(r)+\frac{C}{r} y(r-1) .
$$

Using the induction hypothesis, we know

$$
2 \log y(r) \leq-2\left(\frac{3}{2}\right)^{m} \varepsilon r
$$

From (6.16) and (6.17), it follows that

$$
-\frac{7}{4}\left(\frac{3}{2}\right)^{m} A \varepsilon \mathrm{e}^{-\frac{7}{4}\left(\frac{3}{2}\right)^{m} \varepsilon r} \leq-\left(2\left(\frac{3}{2}\right)^{m} \varepsilon-\frac{C}{r} \mathrm{e}^{\frac{7}{4}\left(\frac{3}{2}\right)^{m} \varepsilon}\right) A \mathrm{e}^{-\frac{7}{4}\left(\frac{3}{2}\right)^{m} \varepsilon r} .
$$

After simplifying this inequality, we get

$$
\frac{1}{4}\left(\frac{3}{2}\right)^{m} \varepsilon \leq \frac{C}{r} \mathrm{e}^{\frac{7}{4}\left(\frac{3}{2}\right)^{m} \varepsilon} \leq \frac{C}{t_{m+1}+1} \mathrm{e}^{\frac{7}{4}\left(\frac{3}{2}\right)^{m} \varepsilon} .
$$

This is not possible if we pick $t_{m+1}$ large enough. Therefore,

$$
y(t)<A \mathrm{e}^{-\frac{7}{4}\left(\frac{3}{2}\right)^{m} \varepsilon t}, \quad \text { for all } t \geq t_{m+1} .
$$


By choosing an even larger $t_{m+1}$ if necessary, we have

$$
y(t)<\mathrm{e}^{-\left(\frac{3}{2}\right)^{m+1} \varepsilon t}, \quad \text { for all } t \geq t_{m+1} .
$$

In other words, (6.15) is true for any $m$. Consequently, for any $a>0$,

$$
y(t) \leq C(a) \mathrm{e}^{-a t}, \quad \text { for all } t
$$

However, by Lemma 6.1, one sees that $y(t)$ decays at most exponentially with a fixed rate. Indeed, we may choose $c(n)>0$ such that the geodesic ball

$$
B_{x}(1) \subset D(t+c(n)+1) \backslash D(t-c(n)-1),
$$

where $d(p, x)=t$. Then, by Lemma 6.1 ,

$$
y(t-c(n)-2) \geq V\left(B_{x}(1)\right) \geq C_{1}(n) V\left(B_{p}(1)\right) \mathrm{e}^{-C_{2}(n) t},
$$

and this contradicts (6.18). This contradiction necessarily implies $\operatorname{Vol}(M)=\infty$. The proof is completed.

We are now ready to prove Theorem 6.1 .

Proof of Theorem 6.1. Recall $D(r):=\{\rho \leq r\}$, where $\rho:=2 \sqrt{f}$. According to $[6]$,

$$
d(p, x)-a \leq \rho(x) \leq d(p, x)+a
$$

with constant $a$ depending only on the dimension $n$. So we may choose $r_{0}>100$ only depending on $n$ such that $D(r)$ has positive measure for $r \geq r_{0}$.

Let $V(t):=\operatorname{Vol}(D(t))$ and $\chi(t):=\int_{D(t)} S$. Then by [6],

$$
\chi(t) \leq \frac{n}{2} V(t)
$$

for any $t>0$. To prove Theorem 6.1, it is sufficient to show that $V(r) \geq C r$, for all $r \geq 2 r_{0}$ for some positive constant $C$ depending only on $n$ and $\mu_{0}$. 
We are going to prove this by contradiction. Let us assume that for $\varepsilon>0$ small depending only on $n$ and $\mu_{0}$ there exists $r \geq 2 r_{0}$ such that

$$
V(r) \leq \varepsilon r .
$$

The choice of $\varepsilon$ will be made clear in the proof. Without loss of generality, we may assume that $r \in \mathbb{N}$. Consider the following set of positive integers:

$$
\Omega:=\{k \in \mathbb{N}: V(t) \leq 2 \varepsilon t, \quad \text { for all integers } r \leq t \leq k\}
$$

Clearly, $r \in \Omega$. We now prove that in fact any integer $k \geq r$ is in $\Omega$, which follows from the following claim.

Claim 6.1. $k+1 \in \Omega$ whenever $k \in \Omega$.

Most of our argument is devoted to proving Claim 6.1. The following logarithmic Sobolev inequality established by Carillo and Ni [7] will again be central to our proof.

$$
\int_{M} u^{2} \log u^{2}-\left(\int_{M} u^{2}\right)\left(\log \int_{M} u^{2}\right) \leq \mu_{0} \int_{M} u^{2}+\int_{M} S u^{2}+4 \int_{M}|\nabla u|^{2}
$$

for any $u \in C_{0}^{\infty}(M)$, where $\mu_{0}$ is the Perelman's invariant. Note that the scalar curvature $S \geq 0$ by $[5,8]$.

For $t \geq 2 r_{0}$, define function $u$ by

$$
u(x)= \begin{cases}1, & \text { on } D(t+1) \backslash D(t), \\ t+2-\rho(x), & \text { on } D(t+2) \backslash D(t+1), \\ \rho(x)-(t-1), & \text { on } D(t) \backslash D(t-1), \\ 0, & \text { otherwise. }\end{cases}
$$

Obviously, $u$ is Lipschitz with compact support. Plugging $u$ into the preceding logarithmic Sobolev inequality and noting that $x \log x \geq-\frac{1}{\mathrm{e}}$ for any $x>0$, we conclude

$$
\begin{aligned}
-\left(\int_{M} u^{2}\right) \log (V(t+2)-V(t-1)) \leq & C_{0}(V(t+2)-V(t-1)) \\
& +(\chi(t+2)-\chi(t-1))
\end{aligned}
$$

where $C_{0}:=\mu_{0}+4+\frac{1}{\mathrm{e}}$. 
On the other hand, according to [6],

$$
\frac{V(t+1)}{(t+1)^{n}}-\frac{V(t)}{t^{n}} \leq 4 \frac{\chi(t+1)}{(t+1)^{n+2}}, \quad \text { for any } t>\sqrt{2(n+2)}
$$

Since $\chi(t) \leq \frac{n}{2} V(t)$, we get from (6.22) that

$$
\begin{aligned}
V(t+1)-V(t) & \leq(t+1)^{n}\left(\frac{V(t)}{t^{n}}+2 n \frac{V(t+1)}{(t+1)^{n+2}}\right)-V(t) \\
& =\frac{(t+1)^{n}-t^{n}}{t^{n}} V(t)+2 n \frac{V(t+1)}{(t+1)^{2}}
\end{aligned}
$$

Observe that

$$
\frac{(t+1)^{n}-t^{n}}{t^{n}} \leq \frac{2^{n}}{t}
$$

So we have

$$
V(t+1)-V(t) \leq 2^{n} \frac{V(t)}{t}+2 n \frac{V(t+1)}{(t+1)^{2}}
$$

In particular, there exists $C(n)$ depending only on $n$ such that

$$
V(t+1) \leq 2 V(t)
$$

for all $t \geq C(n)$.

Plugging this back into (6.23), we find that

$$
V(t+1)-V(t) \leq C_{1} \frac{V(t)}{t}
$$

for all $t \geq C(n)$, where $C_{1}$ depends only on $n$. Moreover, using (6.24) and (6.25) or, alternatively using the same argument as above, we also obtain

$$
V(t+2)-V(t-1) \leq C_{2} \frac{V(t)}{t}
$$

for all $t \geq C(n)$, where $C_{2}$ depends only on $n$. 
Now for all integers $r \leq t \leq k$, note that $t \in \Omega$. So $\frac{V(t)}{t} \leq 2 \varepsilon$, which leads to

$$
\begin{aligned}
V(t+1)-V(t) & \leq 2 C_{1} \varepsilon \\
V(t+2)-V(t-1) & \leq 2 C_{2} \varepsilon .
\end{aligned}
$$

Plugging this into (6.21), we arrive at

$$
\begin{aligned}
(V(t+1)-V(t)) \log \left(2 C_{2} \varepsilon\right)^{-1} \leq & C_{0}(V(t+2)-V(t-1)) \\
& +(\chi(t+2)-\chi(t-1))
\end{aligned}
$$

provided $\varepsilon$ is chosen to satisfy $2 C_{2} \varepsilon<1$.

Iterating (6.26) from $t=r$ to $t=k$ and summing up all the resulting inequalities, we get

$$
(V(k+1)-V(r)) \log \left(2 C_{2} \varepsilon\right)^{-1} \leq 3 C_{0}(V(k+2)+\chi(k+2)) .
$$

Using again that $\chi(k+2) \leq \frac{n}{2} V(k+2)$ and also (6.24), we obtain that

$$
(V(k+1)-V(r)) \log \left(2 C_{2} \varepsilon\right)^{-1} \leq C_{3} V(k+1),
$$

where $C_{3}$ depends only on dimension $n$ and the Perelman's invariant $\mu_{0}$. Rearranging the terms, and using (6.19), we get

$$
\begin{aligned}
V(k+1) & \leq V(r) \frac{\log \left(2 C_{2} \varepsilon\right)^{-1}}{\log \left(2 C_{2} \varepsilon\right)^{-1}-C_{3}} \\
& \leq \varepsilon r \frac{\log \left(2 C_{2} \varepsilon\right)^{-1}}{\log \left(2 C_{2} \varepsilon\right)^{-1}-C_{3}} .
\end{aligned}
$$

Let us choose $\varepsilon$ small enough, depending on $n$ and $\mu_{0}$, so that

$$
\frac{\log \left(2 C_{2} \varepsilon\right)^{-1}}{\log \left(2 C_{2} \varepsilon\right)^{-1}-C_{3}} \leq 2 .
$$

From (6.27) we conclude that

$$
V(k+1) \leq 2 \varepsilon r, \quad \text { for any } k \in \Omega
$$

Since $r \leq(k+1)$, by $(6.28)$ this proves Claim 6.1 . 
We have thus proved that

$$
\Omega=\{k \in \mathbb{N}: k \geq r\}
$$

However, (6.28) now implies that $V(k) \leq 2 \varepsilon r$, for any integer $k \geq r$. This implies that the volume of $M$ is finite, which is a contradiction to Lemma 6.2.

This contradiction indicates there exists no such $r>r_{0}$ such that $V(r) \leq$ $\varepsilon r$ with the $\varepsilon>0$ chosen in the preceding argument, which depends only on $n$ and $\mu_{0}$. That is, $V(r) \geq \varepsilon r$ for $r>r_{0}$. Theorem 6.1 is proved.

\section{Acknowledgment}

The first author has been partially supported by NSF grant no. DMS1005484, and the second author by DMS-1105799.

\section{References}

[1] D. Bakry and M. Émery, Diffusions hypercontractives, in 'Seminaire de probabilites', XIX, 1983/84, Lecture Notes Math., 1123, Springer, Berlin, 1985, pp. 177-206.

[2] P. Buser, A note on the isoperimetric constant, Ann. Sci. Ecole Norm. Sup. 15 (1982), 213-230.

[3] S.Y. Cheng, Eigenvalue comparison theorems and its geometric applications, Math. Z. 143 (1975), 289-297.

[4] E. Calabi, On manifolds with non-negative Ricci curvature II, Notices Amer. Math. Soc. 22 (1975), A205.

[5] H.D. Cao, Geometry of complete gradient shrinking Ricci solitons, in Geometry and Analysis I, Adv. Lect. Math. 17 (2011), 227-246.

[6] H.D. Cao and D. Zhou, On complete gradient shrinking Ricci solitons, J. Differential Geom. 85(2) (2010), 175-186.

[7] J. Carillo and L. Ni, Sharp logarithmic sobolev inequalities on gradient solitons and applications, Comm. Anal. Geom. 17 (2009), 721-753.

[8] B.L. Chen, Strong uniqueness of the Ricci flow, J. Differential Geom. 82(2) (2009), 362-382. 
[9] B. Chow, P. Lu and L. Ni, Hamilton's Ricci flow, Grad. Stud. Math. 77 (2006), 608 pp.

[10] P. Hajłasz and P. Koskela, Sobolev meets Poincaré, C. R. Acad. Sci. Paris Sr. I Math. 320 (1995), 1211-1215.

[11] R. Hamilton, The formation of singularities in the Ricci flow, Surv. Differ. Geom. 2 (1995), International Press, 7-136.

[12] R. Haslhofer and R. Muller, A compactness theorem for complete Ricci shrinkers, Geom. Funct. Anal. 21 (2011), 1091-1116.

[13] P. Li, Harmonic functions and applications to complete manifolds, Lecture Notes on personal web page, available on http:// math.uci.edu/ pli/

[14] P. Li, Lecture notes on geometric analysis, Lect. Notes Ser. 6, Research Institute of Mathematics, Global Analysis Research Center, Seoul National University, Korea, 1993.

[15] P. Li and L-F. Tam, Harmonic functions and the structure of complete manifolds, J. Differential Geom. 35 (1992), 359-383.

[16] P. Li and J. Wang, Complete manifolds with positive spectrum, J. Differential Geom. 58 (2001), 501-534.

[17] P. Li and J. Wang, Complete manifolds with positive spectrum, II, J. Differential. Geom. 62 (2002), 143-162.

[18] P. Li and J. Wang, Connectedness at infinity of complete Kähler manifolds, Amer. J. Math. 131 (2009), 771-817.

[19] P. Li and J. Wang, Weighted Poincaré inequality and rigidity of complete manifolds, Ann. Sci. École. Norm. Sup. (4) 39 (2006), 921-982.

[20] O. Munteanu and J. Wang, Smooth metric measure spaces with nonnegative curvature, Comm. Anal. Geom. 19(3) (2011), 451-486.

[21] G. Perelman, The entropy formula for the Ricci flow and its geometric applications, arXiv:math. DG/0211159.

[22] P. Petersen and W. Wylie, Rigidity of gradient Ricci solitons, Pacific J. Math. 241(2) (2009), 329-345.

[23] D.H. Phong, J. Song, J. Sturm and B. Weinkove, The Kahler-Ricci flow and the $\bar{\partial}$-operator on vector fields, J. Differential Geom. 81(3) (2009), 631-647. 
[24] S. Pigoli, M. Rimoldi and A. Setti, Remarks on non-compact gradient Ricci solitons, Math. Z. 268(3-4) (2011), 777-790.

[25] L. Saloff-Coste, Aspects of Sobolev-type inequalities, London Math. Soc. Lect. Notes Ser. 289 (2002), 190 pp.

[26] N. Sesum and G. Tian, Bounding scalar curvature and diameter along the Kahler Ricci flow (after Perelman), J. Inst. Math. Jussieu 3(3) (2008), 575-587.

[27] Y. Su and H. Zhang, Rigidity of manifolds with Bakry-Émery Ricci curvature bounded below, to appear in Geom. Dedicata.

[28] G. Wei and W. Wylie, Comparison geometry for the Bakry-Émery Ricci tensor, J. Differential Geom. 83 (2009), 377-405.

[29] J.-Y. Wu, Upper bounds on the first eigenvalue for a diffusion operator via Bakry-Émery Ricci curvature II, arXiv:1010.4175.

[30] S.T. Yau, Harmonic functions on complete Riemannian manifolds, Comm. Pure Appl. Math. 28 (1975), 201-228.

[31] S.T. Yau, Some function-theoretic properties of complete Riemannian manifolds and their applications to geometry, Indiana Univ. Math. J. 25 (1976), 659-670.

[32] S. Zhang, On a sharp volume estimate for gradient Ricci solitons with scalar curvature bounded below, Acta Math. Sin. 27(5) (2011), 871-882.

[33] X.-D. Li, Liouville theorems for symmetric diffusion operators on complete Riemannian manifolds, J. Math. Pures. Appl. 84 (2005), 1295-1361.

Department of Mathematics

Columbia University

NEW YORK, NY 10027

USA

E-mail address: omuntean@math.columbia.edu

School of Mathematics

UNIVERSITY OF MINNESOTA

MiNNEAPOLIS, MN 55455

USA

E-mail address: jiaping@math.umn.edu

ReCeived June 19, 2011 